\title{
INFLUENCE DU NOMBRE DE MIRACIDIUMS \\ ET DE L'AGE DU MOLLUSQUE \\ sur la survie et le degré d'infestation \\ de Lymnaea glabra Müller par Fasciola hepatica L.
}

\author{
D. BOUIX-BUSSON*, D. RONDELAUD** et J. PREVOST*
}

RÉSUMÉ. De jeunes Lymnaea glabra ont été exposées aux miracidiums de Fasciola hepatica en tenant compte des deux points suivants : 1) le nombre de miracidiums pour une seule exposition ; 2) la hauteur du mollusque $(0,5 \mathrm{~mm}, 1 \mathrm{~mm}$ ou $2 \mathrm{~mm})$ lors de l'exposition aux miracidiums.

Seules les séries de mollusques hauts de $0,5 \mathrm{~mm}$ ou de $1 \mathrm{~mm}$ lors de l'exposition miracidienne ont présenté des limnées à infestation évolutive au $45^{\mathrm{e}}$ jour postexposition.

Chez les limnées hautes au départ de $0,5 \mathrm{~mm}$, le degré d'infestation montre un pic pour 5 miracidiums par mollusque. Chez les limnées hautes de $1 \mathrm{~mm}$, le degré d'infestation est d'abord nul jusqu'à 5 miracidiums par mollusque, puis il augmente en fonction du nombre de miracidiums utilisés.

Ces résultats montrent que la limnée étudiée est un hôte intermédiaire accidentel pour Fasciola hepatica.

\section{The effects of the number of miracidia and snail height on the survival and infection rate of Lymnaea glabra Müller by Fasciola hepatica $\mathrm{L}$.}

SUMMARY. This report summarizes studies on the infection of Lymnaea glabra by Fasciola hepatica in connection with the following : 1) the number of miracidia for a single exposure ; 2) the snail height $(0,5 \mathrm{~mm}, 1 \mathrm{~mm}$ or $2 \mathrm{~mm})$ at miracidial exposure.

Snails with evolutive infection were only observed in 0,5 - and 1-mm-high groups at day postexposure.

In 0,5-mm-high groups, the percentage of these infected snails showed a peak for 5 miracidia per snail. In 1-mm-high groups, there was no infected snails until 5 miracidia per snail; then the percentage of infected snails increased in connection with the number of miracidia used per snail. L. glabra is an accidental snail host for $F$. hepatica.

\footnotetext{
* Laboratoire d'Écologie et de Biologie Générales (Pr J. Prevost), U.E.R. des Sciences Exactes et Naturelles, 123, rue Albert-Thomas, F 87060 Limoges Cedex.

** Laboratoire d'Histologie (Pr D. Barthe), Faculté de Médecine, 2, rue du Docteur-Raymond-Marcland, F 87032 Limoges Cedex.
}

Accepté le 28 décembre 1982 . 


\section{Introduction}

Diverses espèces de mollusques du genre Lymnaea peuvent assurer le développement des parthenitae de Fasciola hepatica. C'est le cas de L. glabra, de L. palustris, de $L$. peregra, de $L$. stagnalis et de $L$. truncatula pour l'Europe de l'Ouest (Euzeby, 1971). Ces espèces sont des hôtes intermédiaires dans le cycle évolutif de ce parasite.

Un premier rapport signale la présence de $L$. glabra dans les cressonnières à l'origine de cas de distomatose humaine (Rondelaud, 1978). Les jeunes de 21 populations ont été exposés aux miracidiums de $F$. hepatica et par la suite ont présenté une infestation évolutive avec un taux variable (Rondelaud, 1980). Des cercaires ont été obtenues à partir de ces limnées et se sont toutes révélées viables chez l'hôte définitif (Busson et coll., 1982).

Le manque relatif de données sur le rôle de $L$. glabra en tant qu'hôte intermédiaire de $F$. hepatica (Kendall, 1950) et l'abondance de cette limnée dans la région du Limousin (Busson, 1980) ont conduit à préciser les caractéristiques de son infestation fasciolienne. Cette première note a pour but de comparer la survie de L. glabra et son degré d'infestation en fonction du nombre de miracidiums utilisé lors de l'exposition et de l'âge du mollusque.

\section{Matériel et méthodes}

\section{1 - Les séries expérimentales}

Le matériel animal est constitué par 24 séries de mollusques comprenant 21 séries de jeunes soumis à l'exposition aux miracidiums et trois séries de témoins non exposés aux parasites. Chaque série porte sur 1000 jeunes au départ de l'expérimentation.

Ces 24 séries ont été constituées en tenant compte des deux facteurs suivants : 1) La hauteur de la coquille lors de l'exposition aux miracidiums. Les mollusques mesuraient alors : (a) $0,5 \mathrm{~mm}$ (entre 1 et 24 heures de vie); (b) $1 \mathrm{~mm}$ (entre 4 et 8 jours de vie) ; (c) $2 \mathrm{~mm}$ (entre 10 et 15 jours de vie environ). Cette gamme de tailles a été retenue en fonction des résultats obtenus lors d'une étude préliminaire (Busson, 1980). Rappelons que le mollusque adulte présente une hauteur maximale de 18 à $20 \mathrm{~mm}$.

2) Le nombre de miracidiums pour une seule exposition. Chaque limnée est mise en présence de $1,2,5,10,20,50$ miracidiums ou encore d'une masse de 200 à 500 parasites.

Les jeunes proviennent d'un élevage maintenu au laboratoire depuis trois années.

\section{2 - Les techniques utilisées}

Les mollusques ont été élevés par groupes de 50 sur des bacs de $0,12 \mathrm{~m}^{2}$ de surface chacun. Le fond de chaque bac est recouvert par un sédiment calcaro-marneux (argile de décalcification) avec une couche d'eau de 3 à $3,5 \mathrm{~cm}$ d'épaisseur. Cette eau est 
maintenue à niveau constant et soumise à une oxygénation artificielle permanente. Les autres conditions d'élevage sont les suivantes : température de $20^{\circ} \mathrm{C} \pm 1^{\circ} \mathrm{C}$, photopériode naturelle. Des feuilles de salade sont ajoutées deux fois par semaine en complément de la nourriture algale présente dans les bacs.

Les jeunes des 21 séries expérimentales ont été chacun mis en présence du ou des miracidium(s) dans une petite boîte de Pétri de $25 \mathrm{~mm}$ de diamètre avec une couche d'eau de $2 \mathrm{~mm}$ d'épaisseur; la durée de l'exposition est de 4 heures. Les $œ u f s$ de $F$. hepatica utilisés ont été recueillis à l'abattoir local dans la vésicule biliaire de bovins présentant une infestation fasciolienne importante; ils ont été tamisés, lavés et mis en incubation pendant 20 jours à $20^{\circ} \mathrm{C}$ à l'obscurité totale (Ollerenshaw, 1971).

Les mollusques survivants ont été dénombrés dans les diverses séries au $45^{\mathrm{e}}$ jour postexposition. Les rédies et les cercaires se voient alors nettement dans le corps des mollusques grâce à la transparence de la coquille : ceci permet de décompter les limnées à infestation évolutive et celles où l'infestation est abortive parmi les survivants des séries expérimentales.

La survie des limnées à infestation évolutive décroît à partir du $50 \mathrm{e}$ jour postexposition. En effet les mollusques émettent leurs premières cercaires et meurent le plus souvent après.

Les divers résultats ont été traités par la méthode statistique (test $t$ de Student).

\section{Résultats}

Le tableau $I$ indique les résultats obtenus pour les 24 séries : ceux-ci concernent le nombre des survivants et celui des limnées à infestation évolutive. Les degrés d'infestation ont été calculés par rapport aux nombres des survivants.

TABleau I. - Le nombre des survivants (S) et des limnées à infestation évolutive (IE) au 45 jour postexposition. D : degré d'infestation des survivants (en \%). Chaque série comporte 1000 jeunes au départ de l'expérimentation.

\begin{tabular}{|c|c|c|c|c|c|c|c|c|c|}
\hline \multirow{3}{*}{$\begin{array}{l}\text { Nombre de } \\
\text { mira- } \\
\text { cidiums }\end{array}$} & \multicolumn{9}{|c|}{ Hauteur de la coquille lors de l'exposition aux miracidiums } \\
\hline & \multicolumn{3}{|c|}{$0,5 \mathrm{~mm}$} & \multicolumn{3}{|c|}{$1 \mathrm{~mm}$} & \multicolumn{3}{|c|}{$2 \mathrm{~mm}$} \\
\hline & $\overline{\mathrm{S}}$ & IE & $\mathrm{D}$ & $\bar{S}$ & IE & $\bar{D}$ & $\mathrm{~S}$ & IE & $\overline{\mathrm{D}}$ \\
\hline 0 & 765 & - & 一 & 937 & 一 & - & 951 & 一 & - \\
\hline 1 & 566 & 64 & 11,3 & 922 & 0 & 一 & 963 & 0 & - \\
\hline 2 & 556 & 72 & 12,9 & 862 & 0 & - & 941 & 0 & - \\
\hline 5 & 357 & 123 & 34,4 & 910 & 0 & - & 960 & 0 & - \\
\hline 10 & 301 & 32 & 10,6 & 753 & 11 & 1,3 & 919 & 0 & - \\
\hline 20 & 251 & 43 & 17,1 & 481 & 21 & 4,3 & 913 & 0 & - \\
\hline 50 & 99 & 35 & 35,3 & 422 & 63 & 14,9 & 896 & 0 & - \\
\hline masse & 42 & 25 & 59,5 & 311 & 73 & 23,4 & 882 & 0 & - \\
\hline
\end{tabular}




\section{1 - Limnés hautes de $0,5 \mathrm{~mm}$ lors de l'exposition}

Le nombre des survivants n'est que de 763 individus dans la série témoin. Dans les séries expérimentales, on assiste par contre à une diminution de ce nombre depuis la série avec 1 miracidium par limnée jusqu'à celle avec une masse de parasites : les chiffres vont respectivement de 566 à 42 survivants.

Les limnées à infestation évolutive augmentent en nombre depuis la série avec 1 miracidium par mollusque jusqu'à celle avec 5 miracidiums par individu (de 64 à 123 limnées respectivement). Les chiffres sont par contre plus faibles dans les autres séries : ils sont compris entre 25 et 43 unités.

Le degré d'infestation des survivants augmente de même jusqu'à la série avec 5 miracidiums par limnée où il est de $34,4 \%$. Par contre le degré n'est que de 10,6\% dans la série avec 10 miracidiums par mollusque et il augmente par la suite dans les autres séries pour atteindre $59,2 \%$ dans la série exposée à une masse de parasites.

Le test $t$ de Student montre qu'il n'y a pas de différence significative entre les résultats des séries avec 1 et 2 miracidiums par limnée. Par contre les différences entre les degrés d'infestation dans les autres séries sont toutes significatives au seuil de $1 \%$.

\section{2 - Limnées hautes de $1 \mathrm{~mm}$}

Dans la série témoin, 937 survivants ont été dénombrés au 45 e jour postexposition. Dans les séries avec 1, 2 ou 5 miracidiums par limnée, le nombre des survivants décroît faiblement en passant de 922 à 862 individus ; puis il y a une diminution régulière de ce nombre dans les 4 autres séries puisque l'on passe de 753 survivants dans la série avec 10 miracidiums par mollusque à 311 dans la série avec une masse de parasites.

Il n'y a aucune limnée à infestation évolutive dans les séries avec 1, 2 ou 5 miracidiums par mollusque. Des limnées infestées se retrouvent par contre dans les quatre autres séries et leur nombre augmente depuis la série avec 10 miracidiums par mollusque jusqu'à celle qui a été exposée à une masse de parasites (de 11 à 73 limnées respectivement).

Le degré d'infestation des survivants passe de $1,3 \%$ à $23,4 \%$ pour les mêmes séries expérimentales.

Le test $t$ de Student montre que les différences entre les résultats des quatre séries sont toutes significatives au seuil de $1 \%$.

\section{3 - Limnées hautes de $2 \mathrm{~mm}$}

Le nombre des survivants est de 951 chez les témoins. Dans les séries expérimentales, ce nombre diminue depuis la série avec 1 miracidium par mollusque jusqu'à celle qui a été exposée à une masse de parasites (de 963 à 982 survivants respectivement).

Nous n'avons pas retrouvé de limnées à infestation évolutive dans ces séries. 


\section{Discussion}

$\mathrm{Au} 45^{\mathrm{e}}$ jour postexposition, la survie de L. glabra s'accroît de manière régulière avec l'âge des jeunes lors de l'exposition : elle est ainsi de 56,6 \%, 92, $2 \%$ et $96,3 \%$ dans les séries exposées à un seul miracidium avec des mollusques hauts respectivement de $0,5 \mathrm{~mm}, 1$ et $2 \mathrm{~mm}$. Par contre cette survie diminue progressivement lorsque s'accroît le nombre de miracidiums utilisé pour une seule limnée : chez les mollusques hauts de $0,5 \mathrm{~mm}$, elle passe ainsi de $56,6 \%$ à $4,2 \%$ pour un nombre de miracidiums allant de 1 à une masse.

Le nombre des limnées à infestation évolutive et par suite le degré d'infestation des survivants varient en fonction des séries.

- Chez les limnées hautes de $0,5 \mathrm{~mm}$, le nombre des limnées et le degré d'infestation présentent un pic pour 5 miracidiums par mollusque. A partir de 10 miracidiums par mollusque, le nombre des individus infestés reste faible ; par contre le degré d'infestation s'accroît à partir de 50 miracidiums pour une seule limnée.

- Chez les limnées hautes de $1 \mathrm{~mm}$, il n'y a pas d'individus infestés jusqu'à 5 miracidiums par mollusque. Ce nombre augmente par la suite en fonction du nombre de miracidiums. Les mêmes résultats s'observent au niveau du degré d'infestation .

- Il n'y a pas d'individus infestés dans les séries de mollusques hauts de $2 \mathrm{~mm}$.

Ceci sous-entend que le degré d'infestation de L. glabra par $F$. hepatica diminue lorsque l'âge des limnées augmente.

Peu d'auteurs se sont intéressés à l'infestation fasciolienne de L. glabra. Dans les conditions du laboratoire, seul Kendall (1950) signale que cette espèce pouvait s'infester dans ses premiers jours de vie. Les recherches sur le terrain pour retrouver des L. glabra avec une infestation fasciolienne évolutive sont toutes restées négatives (Mackowiak, 1971; Ensergueix, 1975; Rondelaud, 1981). Ce faible nombre de données bibliographiques implique que la comparaison des résultats ne peut se faire qu'avec les données obtenues chez d'autres espèces de limnées (Boray, 1966, 1967, 1969). D'après les notes de cet auteur, l'augmentation du nombre de miracidiums lors de l'exposition a pour conséquence d'augmenter le degré d'infestation ainsi que le taux de mortalité chez les jeunes limnées testées. Nos résultats confirment les observations de Boray, mais il importe de remarquer les trois points suivants :

1) les séries expérimentales et témoins présentent toutes un certain nombre de morts. Cette mortalité est surtout importante chez les individus de $0,5 \mathrm{~mm}$ de hauteur. Il n'est pas possible de préciser la part de la mortalité revenant au parasitisme, à la technique d'élevage ou à un autre facteur : en effet la dissection de ces morts sous loupe binoculaire n'est guère réalisable par suite de leur décomposition rapide ;

2) dans le cas des limnées hautes de $0,5 \mathrm{~mm}$, l'exposition doit comporter un nombre optimal de miracidiums pour que le nombre des individus avec une infestation évolutive soit maximal au $45^{\mathrm{e}}$ jour postexposition. Dans le cadre de ce travail, le nombre est de 5 miracidiums. Une étude complémentaire est encore nécessaire pour déterminer le nombre " idéal " de miracidiums permettant d'obtenir un nombre maximum de limnées à infestation évolutive ; 
3) les L. glabra hautes de $2 \mathrm{~mm}$ n'ont pas présenté d'individus avec une infestation évolutive, malgré le nombre de miracidiums utilisés. L'augmentation du nombre des expositions (2 ou 3) avec 5 à 20 miracidiums par limnée et par exposition s'est également révélée négative (résultats non représentés). Il semble donc exister pour cette espèce une taille limite au-dessus de laquelle les individus ne peuvent s'infester. Ces données contrastent avec les observations faites chez L. peregra par Boray (1969) puisque des individus âgés de 42 à 50 jours et exposés trois fois aux miracidiums ont pu émettre des cercaires. Cette discordance ne peut s'expliquer que par l'espèce de la limnée expérimentée.

Ces rúsultats confirment que $L$. glabra est un hôte intermédiaire accidentel de $F$. hepatica (Busson et coll., 1982) et que son infestation survient dans des conditions très particulières.

\section{BIBLIOGRAPHIE}

Boray J. C. : Studies on the relative susceptibility of some lymnaeids to infection with Fasciola hepatica and F. gigantica and on the adaptation of Fasciola spp. Ann. Trop. Med. Parasitol., I966, 60, II4-124.

Boray J. C. : Host-parasite relationship between lymnaeid snails and Fasciola hepatica. In : "The reaction of the host to parasitism ». N. G. Elwert Universitats- und Verlagsbuchhandlung, Marburg-Lahn, 1967, 132-140.

Boray J. C. : Experimental fascioliasis in Australia. Adv. Parasitol., 1969, 7, 95-210.

Busson D. : Données préliminaires sur l'écologie de Lymnaea glabra L. dans le département de la Haute-Vienne et sur son infestation par Fasciola hepatica L. Mémoire D.E.A. Écol-Ethol., Tours, I980, $60 \mathrm{p}$.

Busson P., Busson D., Rondelaud D., Pestre-Alexandre M. : Données expérimentales sur l'infestation des jeunes de cinq espèces de limnées par Fasciola hepatica L. Ann. Parasitol. Hum. Comp., $1982,57,555-563$.

Ensergueix M. : Contribution à l'étude de l'épidémiologie de la fasciolose à Fasciola hepatica des ovins en Haute-Vienne. Mémoire T.S.A., Prod. Anim., Limoges-Les Vaseix, 1975, 78 p.

EUzEBY J. : Les maladies vermineuses des animaux domestiques et leurs incidences sur la pathologie humaine. Tome II : Maladies dues aux Plathelminthes. $2^{\mathrm{e}}$ fasc. : Trématodes. Livre 1. Vigot Frères, Paris, 1971, 798 p.

Kendall S. B. : Snail hosts of Fasciola hepatica in Britain. J. Helminthol., 1950, 24, 63-64.

Mackowiak F. : Enquête épidémiologique dans un foyer de distomatose hépatique du nord de la France. (A propos de 60 cas). Thèse Doct. Médecine, Lille, I971, nº 96, II I p.

Ollerenshaw C. B. : Some observations on the epidemiology of fascioliasis in relation to the timing of molluscicide applications in the control of the disease. Vet. Rec., I97 I, 88, I52-164.

RONDELAUD D. : Les cressonnières à l'origine de cas de distomatose humaine en Limousin. Êtude des mollusques vecteurs et leur contrôle biologique. Ann. Parasitol. Hum. Comp., 1978, 53, 623-630.

RONDELAUD D. : Données épidémiologiques sur la distomatose humaine à Fasciola hepatica L. dans la région du Limousin, France. Les plantes consommées et les limnées vectrices. Ann. Parasitol. Hum. Comp., 1980, s5, 393-405.

Rondelaud D. : Le contrôle biologique de Lymnaea truncatula Müller. Bilan d'une expérimentation de neuf années en Haute-Vienne, France. Haliotis, I98I, II, 21 3-224. 